\title{
Micromagnetic simulations of thermally activated magnetization reversal of nanoscale magnets
}

\author{
Gregory Brown ${ }^{1,2}$, M. A. Novotny ${ }^{1}$, and Per Arne Rikvold ${ }^{1,2}$ \\ ${ }^{1}$ Supercomputer Computations Research Institute, \\ ${ }^{2}$ Center for Materials Research and Technology, and Department of Physics \\ Florida State University, Tallahassee, Florida 32306-4130
}

(June 24, 2018)

\begin{abstract}
Numerical integration of a stochastic Landau-Lifshitz-Gilbert equation is used to study dynamic processes in single-domain nanoscale magnets at nonzero temperatures. Special attention is given to including thermal fluctuations as a Langevin term, and the Fast Multipole Method is used to calculate dipole-dipole interactions. It is feasible to simulate these dynamics on the nanosecond time scale for spatial discretizations that involve on the order of $10^{4}$ nodes using a desktop workstation. The nanoscale magnets considered here are single pillars with large aspect ratio. Hysteresis-loop simulations are employed to study the stable and metastable configurations of the magnetization. Each pillar has magnetic end caps. In a time-dependent field the magnetization of the pillars is observed to reverse via nucleation, propagation, and coalescence of the end caps. In particular, the end caps propagate into the magnet and meet near the middle. A relatively long-lived defect is formed when end caps with opposite vorticity meet. Fluctuations are more important in the reversal of the magnetization for fields weaker than the zero-temperature coercive field, where the reversal is thermally activated. In this case, the process must be described by its statistical properties, such as the distribution of switching times, averaged over a large number of independent thermal histories.
\end{abstract}

The effect of temperature on the switching behavior of nanoscale magnets can be quite strong when external fields are applied that are just below the zerotemperature coercive threshold. Under these conditions, thermal fluctuations can provide enough energy to take the magnetization of the system over the barrier that prevents it from aligning with the external field [1]. These issues are important for understanding data integrity and high-speed switching in single-domain magnetic applications.

Nanoscale magnets are modeled using the traditional Landau-Lifshitz-Gilbert equation [2],

$$
\frac{d \mathbf{M}_{i}}{d t}=-\frac{\gamma_{0}}{1+\alpha^{2}} \mathbf{M}_{i} \times\left(\mathbf{H}_{\mathbf{i}}-\frac{\alpha}{M_{S}} \mathbf{M}_{i} \times \mathbf{H}_{i}\right),
$$

whereby the microscopic dipoles $\mathbf{M}_{i}$ precess under their individual, locally observed applied fields $\mathbf{H}_{i}$. The universal gyromagnetic factor is $\gamma_{0}=1.76 \times 10^{7} \mathrm{~Hz} / \mathrm{Oe}$, while the material parameters were selected to match those of bulk iron with a saturation magnetization $M_{S}=1700 \mathrm{emu} / \mathrm{cm}^{3}$, exchange length $l_{x}=2.6 \mathrm{~nm}$, and damping parameter $\alpha=0.1$. For the results considered here, these fields are composed of contributions from a uniform field external to the system, the exchange interaction with neighboring dipoles, and dipole-dipole interactions with all of the other dipoles in the system. Calculation of the latter is dramatically accelerated by using a fast multipole algorithm [3]. The numerical models are based on real nanomagnets that have been fabricated recently using scanning microscopy techniques [4].

Thermal effects are incorporated by adding a random contribution to the local field of each spin, as first proposed by W. F. Brown [5] nearly forty years ago.
He calculated the fluctuation-dissipation theorem for a Stratonovich-type [6] noise by considering the FokkerPlanck formulation of the problem. Numerically we implemented this noise using an Itô-type [6] random field. The difference between the Stratonovich and Itô paradigms does not affect the results since we normalize the dipoles to a fixed length after each integration step.

The individual magnets, rectangular with dimensions $9 \mathrm{~nm} \times 9 \mathrm{~nm} \times 150 \mathrm{~nm}$, were implemented using a finitedifference approach. The numerical results have been found to be independent of discretization for the spatial discretization of $\Delta x=1.5 \mathrm{~nm}$ and integration step of $\Delta t=50$ fs used here.

The large shape anisotropy of these magnets causes spontaneous alignment of the magnetization, except at the top and bottom where pole avoidance leads to the formation of end caps. As can be seen in Fig. 1(a), when the external field is applied in the opposite direction during a hysteresis measurement, the $z$-component of the magnetization is reduced via growth of these end caps. Here the uniform applied field points down, light shades indicate upward-pointing magnetization, and dark shades indicate downward-pointing magnetization. At zero temperature the end caps grow symmetrically; when they meet at the middle of the pillar a relatively long-lived defect is formed due to the opposite helicities of the two end caps. These results are fully consistent with the $T=0$ simulations of Ref. [7]. There the field was swept quasistatically as opposed to the truly dynamic sweeps considered here.

The corresponding hysteresis loop, with a period of $1 \mathrm{~ns}$, is shown in Fig. 2. The defect is indicated by slow decay of the magnetization around the time when the ex- 
ternal field reverses. The effect of this defect is also apparent for the longer-period hysteresis curves, for which the defect disappears before the field reverses. The simulated zero-temperature hysteresis loops are very reproducible, and no differences are seen for subsequent periods. The inset shows example variations that occur from thermal effects for the 1 ns hysteresis loop. The largest differences are seen in conjunction with the defect that forms from the two end caps.

The simplest magnetization-reversal situation to study is one in which the external field suddenly changes its orientation, and then remains constant. In these simulations, the field is initially zero and then is brought negative in $0.25 \mathrm{~ns}$ with its amplitude described by $1 / 4$ of a sine wave. In what follows we set $t=0$ at the time when the field first reaches its maximum negative value. When the final field is less than the coercive field, the magnetization remains oriented upward until thermal fluctuations take it over the associated free-energy barrier. In long pillars the end caps do not interact strongly, and the free energy of each can be considered separately. The free energy as a function of end cap volume has essentially three extrema: one local minimum corresponding to a small end cap, one local maximum corresponding to an unstable volume where the tendency for shrinkage is equal to the tendency for growth, and the global minimum corresponding to a switched magnet (spanning end cap). Snapshots from the magnetization reversal of one magnet in this situation at $H=-1850$ Oe and $20 \mathrm{~K}$ are shown in Fig. 1(b). Here the lower end cap undergoes a large fluctuation to take it past the critical volume, after which it grows at an almost constant rate to fill the entire magnet. (Again a long-lived defect forms when the two end caps come into contact.)

The thermally-activated nature of the end cap growth leads to a distribution in the switching times, defined as the time when $M_{z}=0$. The probability of not switching, $P_{\text {not }}(t)$, for 85 switches at $H=-1800$ Oe and $20 \mathrm{~K}$ is shown as the heavy, stepped curve in Fig. 3. Under these conditions, the majority of simulated switches occur between 0.5 and $1.2 \mathrm{~ns}$ after the field reversal finishes. There are no switches before $0.4 \mathrm{~ns}$ because it takes this amount of time for a single supercritical end cap to grow to fill half of the magnet. The traces of the average pillar magnetization density as a function of time are shown for five different switches in the inset of Fig. 3. There are essentially two slopes observed during the actual switching process, corresponding to cases where one or both end caps are growing.

The observation that the end caps decay essentially independently and exponentially, with rate $\rho$, and that freely growing end caps change the global magnetization (normalized to lie between \pm 1 ) at a constant rate $v$, can be used to construct a simple model to describe the distribution of switching times observed in individual experiments. The resulting probability of not switching is

$$
P_{\text {not }}(t)=\left\{\begin{array}{lr}
1 & t<1 /(2 v) \\
e^{-(2 \rho t-\rho / v)}(1+2 \rho t-\rho / v) & 1 /(2 v) \leq t<1 / v \\
e^{-(2 \rho t-\rho / v)}(1+\rho / v) & 1 / v \leq t
\end{array} .\right.
$$

Taking $\rho$ and $v$ as parameters, a nonlinear fit of this twoexponential decay theory to the first two moments of the data is shown as the dashed line in Fig 3. For comparison, a similar fitting has also been performed for an error function form with two parameters (corresponding to a Gaussian histogram of switching times), which is shown as the dotted curve in Fig. 3. From the 85 switches presented here there is no clear advantage to either fitting function. Different combinations of field and temperature should probe regions where the fitting functions are not so similar.

In summary, Landau-Lifshitz-Gilbert dynamics have been simulated for three-dimensional models of singledomain nanoscale magnets of large aspect ratio. Hysteresis-loop and field-reversal simulations show that magnetization reversal occurs through the nucleation, growth, and coalescence of the end caps. For fieldreversal simulations at nonzero $T$ which require thermal fluctuations to complete the reversal, a simple theory that considers the nucleation rate and growth velocity of the end caps adequately describes the statistical distribution of the switching times.

Supported by NSF grant No. DMR-9871455, NERSC, and by FSU/SCRI and FSU/MARTECH.

[1] P. A. Rikvold, M. A. Novotny, M. Kolesik, and H. L. Richards, Dynamical Properties of Unconventional Magnetic Systems, edited by T. Skjeltorp and D. Sherrington (Kluwer, Dordrecht, 1998)

[2] A. Aharoni, Introduction to the Theory of Ferromagnetism (Clarendon, Oxford, 1996).

[3] L. Greengard and V. Rokhlin, J. Comp. Phys. 73, 325 (1987).

[4] S. Wirth, M. Field, D. D. Awschalom, and S. von Molnár, Phys. Rev. B 57, R14028 (1998).

[5] W. F. Brown, Phys. Rev. 130, 1677 (1963).

[6] N. G. van Kampen, Stochastic Processes in Physics and Chemistry: Revised and enlarged edition (Elsevier, Amsterdam, 1992).

[7] Y. D. Yan and E. Della Torre, J. Appl. Phys. 66, 320 (1989). 
a)

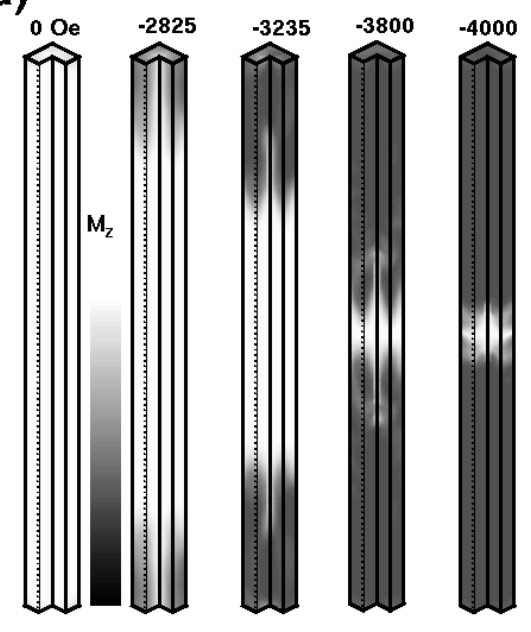

b)

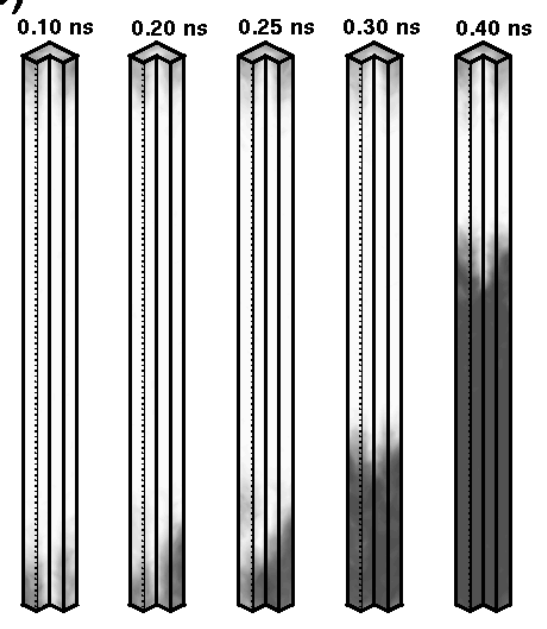

FIG. 1. Visualization of the $z$-component of the magnetization for $9 \mathrm{~nm} \times 9 \mathrm{~nm} \times 150 \mathrm{~nm}$ iron nanomagnets (shown in $3 / 4$ cut-away view). Light shades indicate upward-pointing magnetization, while dark shades indicate downward-pointing magnetization. (a) Switching via symmetric growth of end caps for 1 ns period hysteresis loop at 0 K. (b) Switching at $20 \mathrm{~K}$ and $H=1850$ Oe via thermal fluctuations of one end cap over the saddle point, with subsequent growth to switch the magnetization. Movies are available at http://www.scri.fsu.edu/ browgnrg/micromag/pillar.html

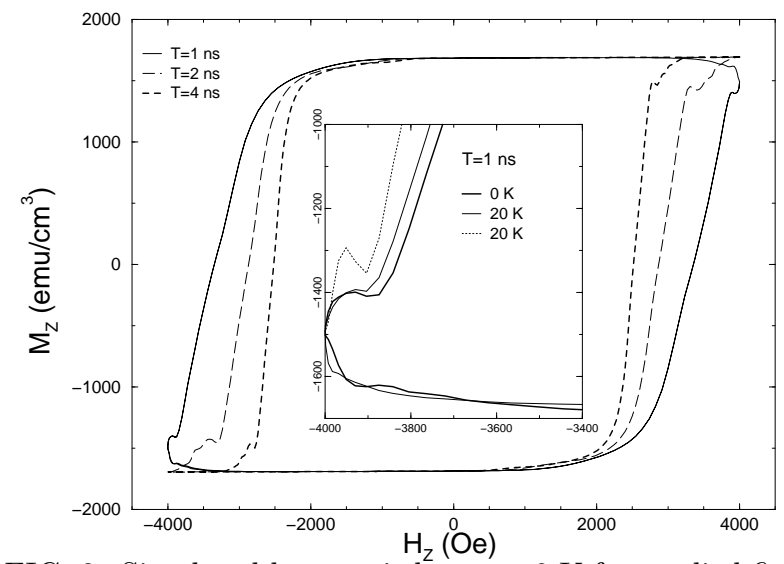

FIG. 2. Simulated hysteresis loops at $0 \mathrm{~K}$ for applied fields oscillating with periods of 1,2 , and 4 ns. The inset shows variations caused by temperature for two loops simulated at $20 K$. The feature that occurs at nearly saturated magnetizations is due to a defect that forms when the end caps come in contact.

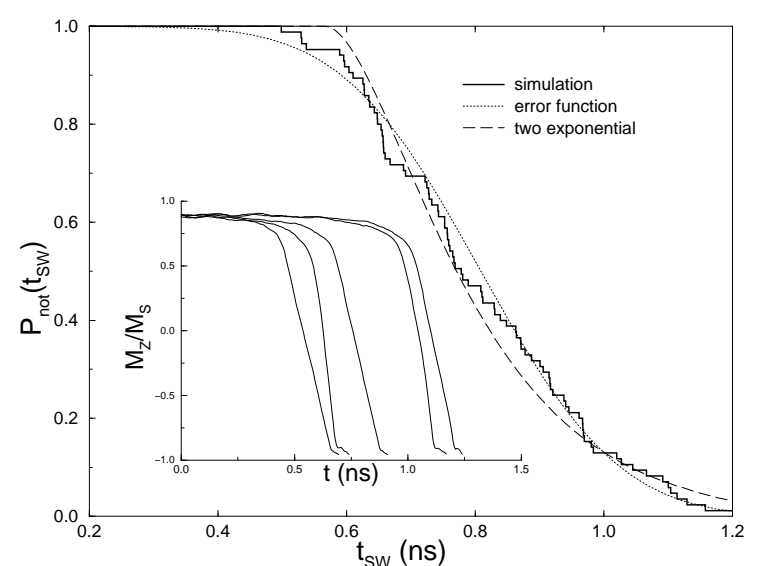

FIG. 3. Probability of not switching, $P_{\text {not }}$, for nanomagnets experiencing external fields just below the coercive value. The solid line is simulation data from 85 switches at $H=-1800$ Oe and $20 \mathrm{~K}$. Two theoretical forms [the dotted line is an error function corresponding to a Gaussian histogram of switching times, and the dashed line corresponds to the two-exponential decay theory described in the text, Eq. (2)] are compared after nonlinear fitting with two parameters. The inset shows 5 examples of the average $z$-component of the magnetization during different switching events. 